\title{
Efeito da experiência do treinador sobre o ambiente motivacional e pedagógico no treino de jovens
}

CDD. 20.ed. 796.011

796.073

\author{
Carlos Eduardo GONÇALVES* \\ Manuel João COELHO E SILVA* \\ Jaume CRUZ ${ }^{* *}$ \\ Antonio FIGUEIREDO*
}

*Faculdade de Ciên-
cias do Desporto e
Educação Física, Uni-
versidade de Coimbra
Portugal.
**Facultat de Psiclogia,
Universitat Autonoma
de Barcelona - Espa-
nha.

\section{Resumo}

0 estudo investiga o efeito da experiência do treinador sobre as categorias observadas da comunicação em competição, as orientações para a realização de objetivos, as atitudes face à prática desportiva e as percepções dos atletas sobre o comportamento do treinador. Utilizando o Coach Behavior Assessment System (CBAS), de SMiTH, SMOLl e Hunt (1977), foram gravados em áudio e vídeo e posteriormente analisados 18 jogos de seis treinadores de Basquetebol do escalão de sub-16 anos masculinos, três experientes e três inexperientes. Os jogadores das equipes observadas $(n=58)$ preencheram as versões portuguesas do Task and Ego Orientation in Sport Questionnaire/TEOSO (CHI \& DUDA, 1995) e do Sport Attitudes Questionnaire/SAQ (LeE \& WHITEHEAD, 1999), bem como o questionário de percepções do comportamento do treinador do CBAS. Os resultados revelaram um efeito estatisticamente significativo da experiência ou inexperiência do treinador, tanto sobre as categorias comportamentais observadas em jogo como sobre as dimensões dos questionários.

UnITERMOS: Jovens; Treinador; Tarefa; Ego; Atitudes.

\section{Introdução}

A configuração do ambiente em que se desenrola a atividade é produto de vários fatores e de agentes socializadores diversos, que podem ir dos adultos próximos - famílias, treinadores - aos pares companheiros de equipe. Os agentes socializadores podem representar para os jovens atletas fontes de informações pertinentes, apoios motivacionais, afetivos e cognitivos e fontes de pressão para a obtenção de resultados (BRUSTAD \& PARTRIDGE, 2002; GREENDORFER, LeWKo \& Rosengren, 2002). Na adolescência, em especial a partir dos 12 anos de idade, a figura do treinador atinge uma importância decisiva, chegando a sobrepor-se à influência das famílias (LeE, 1996; Weigand, Carr, Petherick \& Taylor, 2001).

O papel decisivo do treinador na criação e manutenção do clima de treino e competição é mediado pelas percepçõos dos atletas, que interpretam de modo diverso os comportamentos, atitudes e estilos de liderança dos treinadores (CRUZ, BoIXADÓs, VAliente, Torregrosa \& Mimbrero, 1996; Gilbert \& Trudel, 2004; Penney, 2006; Smoll \& Smith,
2002). As percepções das condutas dos treinadores por parte dos atletas são mais precisas a partir da adolescência e atravessam os vários níveis competitivos e os vários contextos, com impacto sobre a motivação e as orientações dos praticantes.

Estudos anteriores (Jones \& STANDAGE, 2006; JONES \& WALLACE, 2006) realçaram a importância da experiência do treinador na fundamentação e racionalização das decisões tomadas, com particular incidência no relacionamento interpessoal com os atletas e na promoção de ambientes de prática com impacto na qualidade das experiências dos atletas (SChempp, McCullick \& Sannen Mason, 2006). Os mesmos autores afirmam que o treinador inexperiente apresenta dificuldades na escolha dos meios para promover o melhor clima para o aperfeiçoamento do praticante e está excessivamente preocupado em manter o controle de tudo o que se passa no treino e na competição. É geralmente aceitável que o treinador inexperiente é aquele que possui menos de três anos no exercício da função (SCHempp, McCullick \& 
SANNEN MASON, 2006). Interessa pois investigar se a variável experiência do treinador representa um efeito significativo sobre a ecologia da prática desportiva.

Uma das metodologias utilizadas para avaliar a conduta dos treinadores de jovens em treino e competição é a observação direta e o registro, mediante a codificação adequada dos conteúdos resultantes das açōes e discurso dos treinadores. O Sistema de Avaliação de Conduta de Treinadores (Coach Behavior Assessment System, CBAS), de SMith, SMOll e Hunt (1977) permite observar e analisar a comunicação verbal dos treinadores com os atletas mediante o uso de registros áudio-visuais apropriados. O CBAS, sustentado por estudos preliminares, parte do pressuposto que os comportamentos dos treinadores se dividem em dois grandes grupos: a) reativos, quando se traduzem em respostas imediatas a ações de um atleta ou de toda a equipe; b) espontâneos, quando resultam de iniciativa do treinador não relacionada com uma ação precedente.

Smith, Smoll e Hunt (1977) identificam 12 categorias de comunicação, das quais seis são dirigidas ao atleta individualmente e integram três dimensōes comportamentais dos treinadores: a) Sustentadora, que engloba 1) reforço positivo e 2) ânimo ao erro; b) Instrutiva, com 3) instrução técnica geral e 4) instrução técnica ao erro; c) Punitiva, integrando 5) punição e 6) instrução técnica punitiva.

Das restantes seis categorias do CBAS, duas refletem a indiferença do treinador face a ações, positivas ou negativas, do atleta: 7) não reforço; 8) ignorar o erro. As outras quatro exprimem comunicação direcionada para a equipe ou para outros presentes: a) iniciativas destinadas a prevenir ou sanar episódios de disciplina: 9) manter o controle; b) intervençōes para encorajar a equipe: 10) ânimo geral; c) intervençōes para melhorar ou alterar a organização da equipe: 11) organização geral; d) comunicação direcionada a não participantes na competição e não se relacionando com esta: 12) comunicação geral. A observação do treinador pode ser complementada por um questionário que procura discernir as percepções dos atletas sobre os comportamentos do seu treinador. $\mathrm{O}$ instrumento CBAS possui 11 itens, correspondentes às categorias observadas.

O reconhecimento da importância do treinador na promoção do ambiente desportivo, conduz à necessidade de avaliar o ambiente do ponto de vista moral. Vários autores utilizam o termo desportivismo (Boixadós, Cruz, Torregrosa, \& Valiente, 2004; Lee, Whitehead, \& Balchin, 2000; Vallerand, Deshaies, Courrier, Brière, \& Pelletier, 1996), com base em elementos normativos/convencionais, supostamente suportados pelos regulamentos desportivos, tanto cognitivos ("renúncia à vitória a todo o custo", ou "dar o melhor"), como sociais ("dar-me bem com todos os participantes").

Com base nestes pressupostos, LeE e WHITEHEAD (1999) elaboraram o Sports Attitudes Questionnaire - SAQ, composto de 23 itens que foi aplicado a uma amostra de atletas, masculinos e femininos, com idades entre os 13 e os 16 anos, praticantes dos desportos mais populares em Inglaterra. Este estudo resultou em proposta de modelo de quatro fatores, dois dos quais considerados socialmente positivos (compromisso e convenção) e os outros dois socialmente negativos (batota e anti-desportivismo). A consistência interna do modelo de quatro fatores surge como elevada; o alfa de Cronbach para o compromisso foi 0,68 , para a convenção 0,82 , para a batota 0,81 e para o anti-desportivismo 0,84.

A literatura (DUDA, 2001; RoBERTS, 2001) refere a Teoria para a Realização de Objetivos (NICHOLLs, 1989) como um sólido enquadramento teórico para explicar as relaçōes entre as orientaçōes disposicionais e as questōes morais no desporto de jovens. O Questionário de Orientação para a Tarefa e para o Ego -TEOSQ (CHI \& DUDA, 1995) representa um instrumento psicométricamente fiável (HANRAHAN \& BIDDLE, 2002), com satisfatória validação transcultural (Gonçalves, Coelho e Silva \& CRuZ, 2006; Peiró \& Sanchis, 2004). Investigações anteriores demonstraram que as orientações individuais para a tarefa ou para o ego podem ser afetadas por variáveis situacionais criadas por adultos significantes e pares (GREENDORFER, LEWKO \& ROSENGRen, 2002; Magyar \& Felz, 2003; Ntoumanis \& Vazou, 2005; Treasure, 2001; Vazou, Ntoumanis \& DUDA, 2005).

Importa pois investigar se o ambiente de treino e de competição promovido pelo treinador e mediado pela sua experiência profissional exerce efeito sobre o termo Desportivismo, avaliado pelo SAQ, e Orientação para a Realização de Objetivos, avaliado pelo TEOSQ. Para o presente estudo foi escolhida a modalidade desportiva Basquetebol, dada a disponibilidade das organizações e dos treinadores e a regularidade e equilíbrio das competições. Estudos anteriores na modalidade (Coelho e Silva, Rocha \& GonÇalves, 2006) evidenciaram que a maior parte dos treinadores de jovens são também eles muito jovens e com valores muito elevados de atrito e abandono. É importante evidenciar que, mais do que investigar as diferenças de contextos, é necessário compreender as diferenças e semelhanças entre treinadores em contextos desportivos similares (WeRTHNER \& TRUDEL, 2006). 
O presente estudo visa: a) observar e analisar a comunicação de treinadores de Basquetebol de atletas masculinos sub-16 anos em situação de competição, utilizando o Coach Behavior Assessment System/CBAS; b) testar a hipótese de que a experiência dos treinadores

\section{Métodos}

\section{Participantes}

\section{Treinadores}

A amostra de treinadores foi constituída por seis elementos, federados na modalidade de Basquetebol, do escalão de sub-16 anos masculinos e que foram divididos em dois subgrupos, experientes e inexperientes. Estes últimos não poderiam ter uma carreira de preparação e orientação de equipes superior a dois anos. Tendo em conta os regulamentos da Federação Portuguesa de Basquetebol, os treinadores inexperientes eram também aqueles com o grau inferior de formação curricular, escalonada em três níveis. vai exercer efeito significativo sobre as Atitudes face à Prática Desportiva, sobre as Orientaçôes para a Realização de Objetivos e sobre as Percepçôes do Comportamento do Treinador expressas pelos atletas das equipes orientadas pelos treinadores observados.

TABELA 1 - Características dos treinadores experientes.

\begin{tabular}{lccc}
\hline & Treinador A & Treinador B & Treinador C \\
\hline Idade & 44 & 42 & 53 \\
Habilitações acadêmicas & $12^{\circ}$ ano & $12^{\circ}$ ano & $11^{\circ}$ ano \\
Habilitações desportivas & Nível II & Nível II & Nível II \\
Anos de carreira como jogador & 6 & 12 & 20 \\
Nível máximo como jogador & Júnior & $3^{\text {a divisão }}$ & Profissional \\
Anos de carreira como treinador & 24 & 23 & 15 \\
Nível máximo como treinador & $2^{a}$ divisão & Júnior & Profissional \\
Anos no atual escalão & 3 & 10 & 1 \\
\hline
\end{tabular}

As habilitações desportivas dos treinadores são conferidas pela Federação Portuguesa de Basquetebol e estruturam-se em três níveis: o nível I, que habilita para treinar equipes com idades até 16 anos, o nível II, que permite treinar equipes juniores e seniores e o nível III que dá acesso ao treino de equipes de alto rendimento.

O subgrupo de treinadores experientes apresenta homogeneidade na idade, habilitaçôes acadêmicas e desportivas e nos anos de carreira como treinadores.
A seleção dos participantes foi realizada através de consulta a associaçôes distritais da modalidade de Basquetebol, que indicou os treinadores que respondiam aos critérios de participação no estudo e forneceu os respectivos contatos. Posteriormente, o investigador comunicou com os dirigentes dos clubes e com os treinadores, a quem explicou os objetivos da pesquisa e solicitou a autorização do clube e a concordância do treinador. $\mathrm{O}$ investigador principal reuniu separadamente com cada um dos treinadores, que responderam a perguntas relacionadas com dados pessoais e profissionais relevantes para o estudo. As características demográficas da amostra são apresentadas nas TABELAS 1 e 2.
$\mathrm{O}$ treinador $\mathrm{C}$ apresenta um percurso diferente dos outros, com uma carreira de jogador e de treinador que atingiu o topo de rendimento nacional, ao contrário dos treinadores A e B, que centraram as suas carreiras nos escalóes etários mais jovens e em níveis de competição inferiores. Os treinadores inexperientes constituem um subgrupo homogêneo e apenas diferem nas habilitações acadêmicas, que vão desde a escolaridade obrigatória até à licenciatura. 
TABELA 2 - Características dos treinadores inexperientes.

\begin{tabular}{|c|c|c|c|}
\hline & Treinador D & Treinador E & Treinador $\mathbf{F}$ \\
\hline Idade & 32 & 23 & 26 \\
\hline Habilitações acadêmicas & $6^{\circ}$ ano & $11^{\circ}$ ano & Licenciado \\
\hline Habilitações desportivas & Nível I & Nível I & Nível I \\
\hline Anos de carreira como jogador & 1 & 4 & 3 \\
\hline Nível máximo como jogador & $3^{\text {a }}$ divisão & Júnior & Júnior \\
\hline Anos de carreira como treinador & 2 & 2 & 2 \\
\hline Nível máximo como treinador & Sub 16 & Sub 16 & Sub 16 \\
\hline Anos no atual escalão & 1 & 1 & 1 \\
\hline
\end{tabular}

Jovens atletas

sob supervisão dos treinadores observados

Foram inquiridos 58 jogadores praticantes de Basquetebol federado no escalão de sub-16 anos masculinos e integrantes das equipes orientadas pelos treinadores participantes no estudo. Dos 58 jogadores, 31 integravam as equipes supervisionadas por treinadores experientes e 27 das equipes supervisionadas por treinadores inexperientes. Foram considerados apenas os jogadores que participavam regularmente nos treinos e nos jogos da equipe.

\section{Instrumentos}

Foram aplicados aos atletas os seguintes questionários:

Task and Ego Orientation in Sport Questionnaire (TeosQ, Chi \& DudA, 1995). A versão portuguesa do questionário foi obtida por FonseCA e BIDDLE (1996) e validada para a população alvo por GoNçalves, Cardoso, Freitas, Lourenço e Coelho e SiLVA (2005). As respostas aos 13 itens foram dadas em escala de Likert de 1 a 5, em que 1) discordo totalmente, 2) discordo, 3) não concordo nem discordo, 4) concordo, 5) concordo totalmente.

Sport Attitudes Questionnaire (Questionário de Atitudes face ao Desporto/SAQ). As atitudes face ao desporto foram avaliadas com recurso ao questionário de LeE e WhiteheAd (1999). O instrumento é composto de 23 itens que pretendem identificar a opinião dos respondentes sobre o modo como praticam o seu desporto principal. Os participantes responderam em escala de Likert de 1 a 5 , em que 1) discordo totalmente, 2) discordo, 3) não concordo nem discordo, 4) concordo, 5) concordo totalmente. A versão portuguesa foi traduzida e validada por GONÇALVES, Coelho e Silva, Cruz, Lee e Chatzisarantis (2006).
Coach Behavior Assessment System (CBAS), de SMITH, SMOLL e HunT (1977). A versão portuguesa foi obtida a partir da versão castelhana. Esta versão foi obtida através de tradução por dois peritos em Ciências do Desporto com muito bons conhecimentos de inglês, seguida de retroversão por um tradutor cuja língua materna era o inglês. Os participantes responderam aos 11 itens do questionário em escala de Likert de 1 a 5 , em que 1) discordo totalmente, 2) discordo, 3) não concordo nem discordo, 4) concordo, 5) concordo totalmente. Os respondentes foram os jogadores das equipes observadas e os itens do questionário correspondem às categorias observadas.

\section{Procedimentos}

Foram gravados 18 jogos, três por treinador, da fase regular de um campeonato organizado pela $\mathrm{Fe}$ deração Portuguesa de Basquetebol. Os jogos foram registados em vídeo, com recurso a Câmara de vídeo Sony DCR-SR 190 e as intervençōes do treinador registadas mediante a utilização de microfone remoto e receptor Sennheiser SG301. Posteriormente as gravações foram transcritas para $\mathrm{CD}$, de modo a serem visionadas em computador e analisadas.

O número de três jogos observados por treinador, que seguiu as indicações de CRUZ (2003), destinou-se a minimizar as particularidades inerentes a jogos com adversários, resultados ou incidências específicas e a revelar um padrão habitual de intervenção do treinador. Estudos precedentes (CRUZ, 2003; SOUSA, CRUZ, TORREGrosa, Vilches \& VILADRICH, 2006) adotaram a mesma estratégia de acumulação de três unidades e observação, isto é, três jogos por cada treinador em estudo, para conferir robustez relativamente a padróes de intervenção atribuíveis ao acaso.

As gravações de cada um dos jogos foram analisadas por dois observadores diferentes e os dados 
registados em grelha de observação própria, correspondente às 12 categorias do CBAS. Nas células reservadas a cada categoria registaram-se o número de episódios por jogo. Os observadores participaram em duas sessōes de formação, orientadas pelo investigador principal, de modo a uniformizar critérios e esclarecer eventuais dúvidas sobre a interpretação dos episódios observados.

A congruência entre observadores foi avaliada através do indice $k$ de Cohen (CoHen, 1960), referido na literatura como o mais utilizado na análise de variáveis categóricas (LANDIs \& KoCH, 1977). $\mathrm{O}$ indice $k$ indica o grau de concordância existente entre observadores, para além do esperável devido ao acaso. De acordo com Landis e KoCH (1977), os valores de $k$ situados entre 0,41 e 0,60 denotam uma concordância entre observadores moderada, enquanto que a concordância se pode considerar boa quando os valores de $k$ se situam entre 0,61 e 0,80.

Após a aprovação do estudo pelo Comité de Ética competente e obtido o consentimento dos encarregados de educação dos atletas para a sua participação no estudo, o TEOSQ e o SAQ, que procuram discernir dados disposicionais, foram aplicados no início de

\section{Resultados}

Os resultados são apresentados graficamente nas FIGURAS 1 e 2, o primeiro gráfico mostrando as percentagens correspondentes a cada categoria do CBAS por cada um dos seis treinadores observados. A FIGURA 2 mostra as percentagens correspondentes às categorias do CBAS por sub-grupos de treinadores, experientes e inexperientes. Apesar das diferenças individuais, todos os treinadores convergem em privilegiar as categorias reforço, instrução técnica gerale ânimo geral como as que merecem maior ocorrência de episódios. De notar que apenas estas três categorias apresentam percentagens de ocorrências totais superiores a 10. Em termos absolutos, independentemente da experiência, os treinadores realizam cerca de 200 intervençōes por jogo, resultando uma média de 1 intervenção em cada 15 segundos. sessões de treino, na semana seguinte à gravação do primeiro jogo do respectivo clube. O CBAS, que pressupóe um conhecimento aprofundado do treinador por parte dos jogadores, foi aplicado no início de sessões de treino, na semana seguinte à gravação do último jogo do respectivo clube, o que correspondeu a sensivelmente seis meses após o início da época desportiva.

\section{Tratamento de dados}

$\mathrm{Na}$ análise das grelhas de observação de jogos, foram consideradas as ocorrências por categoria e as percentagens do total de ocorrências que cabem a cada categoria. Para o tratamento dos dados resultantes da aplicação dos questionários TEOSQ, SAQ e CBAS e, em particular para testar o efeito da experiência do treinador sobre as respostas dos atletas, foi aplicada estatística descritiva e t-teste. Para testar o efeito da variável independente experiência do treinador sobre as variáveis dependentes dimensões extraídas dos referidos instrumentos, foi utilizada a técnica ANOVA de análise multivariada da variância. Os tratamentos foram efetuados com recurso ao programa SPSS, versão 14.0.

Os dois grupos de treinadores focam as suas intervenções durante o jogo em três categorias: reforço, instrução técnica geral e ânimo geral. Resultados semelhantes são encontrados por CRUZ (2001), com uma amostra de treinadores espanhóis de Basquetebol. Entre os treinadores experientes, as categorias mais frequentes são, por esta ordem, instrução técnica geral, ânimo geral e reforço. Por sua vez, os treinadores com menor experiência recorrem com maior frequência à instrução técnica geral, seguida de reforço e só depois ânimo geral. As categorias ânimo ao erro, punição, instrução técnica punitiva, organização geral e comunicação geral parecem ter valor residual, com frequências relativas quase sempre inferiores a 5\%. 
$R$ (reforço); NR (não reforço); $A E$ (ânimo ao erro) ITE (instrução técnica ao erro);

P (punição);

ITP (instrução técnica punitiva);

IE (ignorar o erro);

$\mathrm{MC}$ (manter o controle);

ITG (instrução técnica geral);

AG (ânimo geral);

OG (organização geral); CG (comunicação geral).

R (reforço);

NR (não reforço);

AE (ânimo ao erro);

ITE (instrução técnica

ao erro);

$P$ (punição);

ITP (instrução técnica punitiva);

IE (ignorar o erro);

MC (manter o controle);

ITG (instrução técnica geral);

AG (ânimo geral);

OG (organização geral):

CG (comunicação geral).

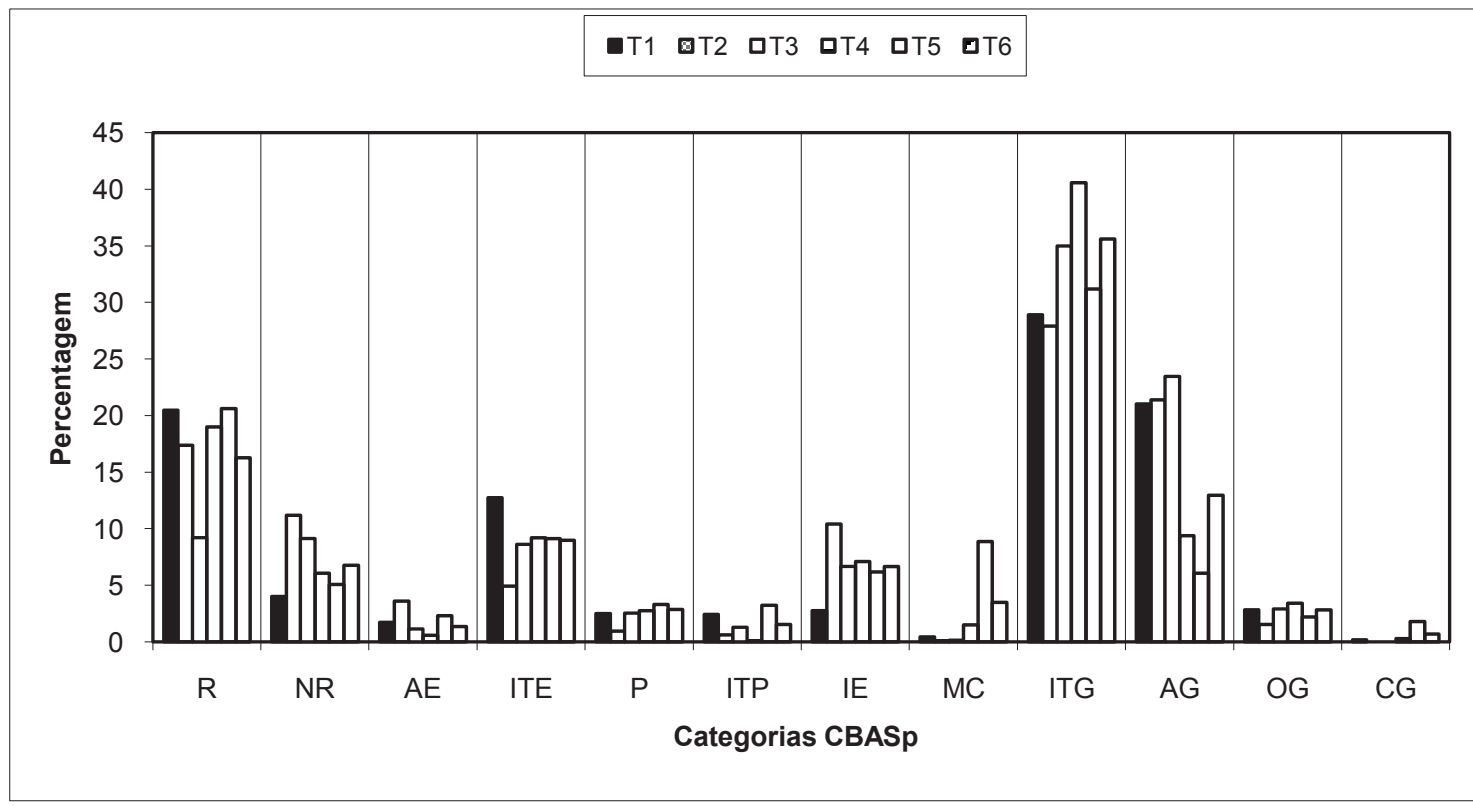

FIGURA 1 - Percentagem de ocorrências nas categorias do Coach Behavior Assessment System (CBAS) para cada um dos treinadores observados.

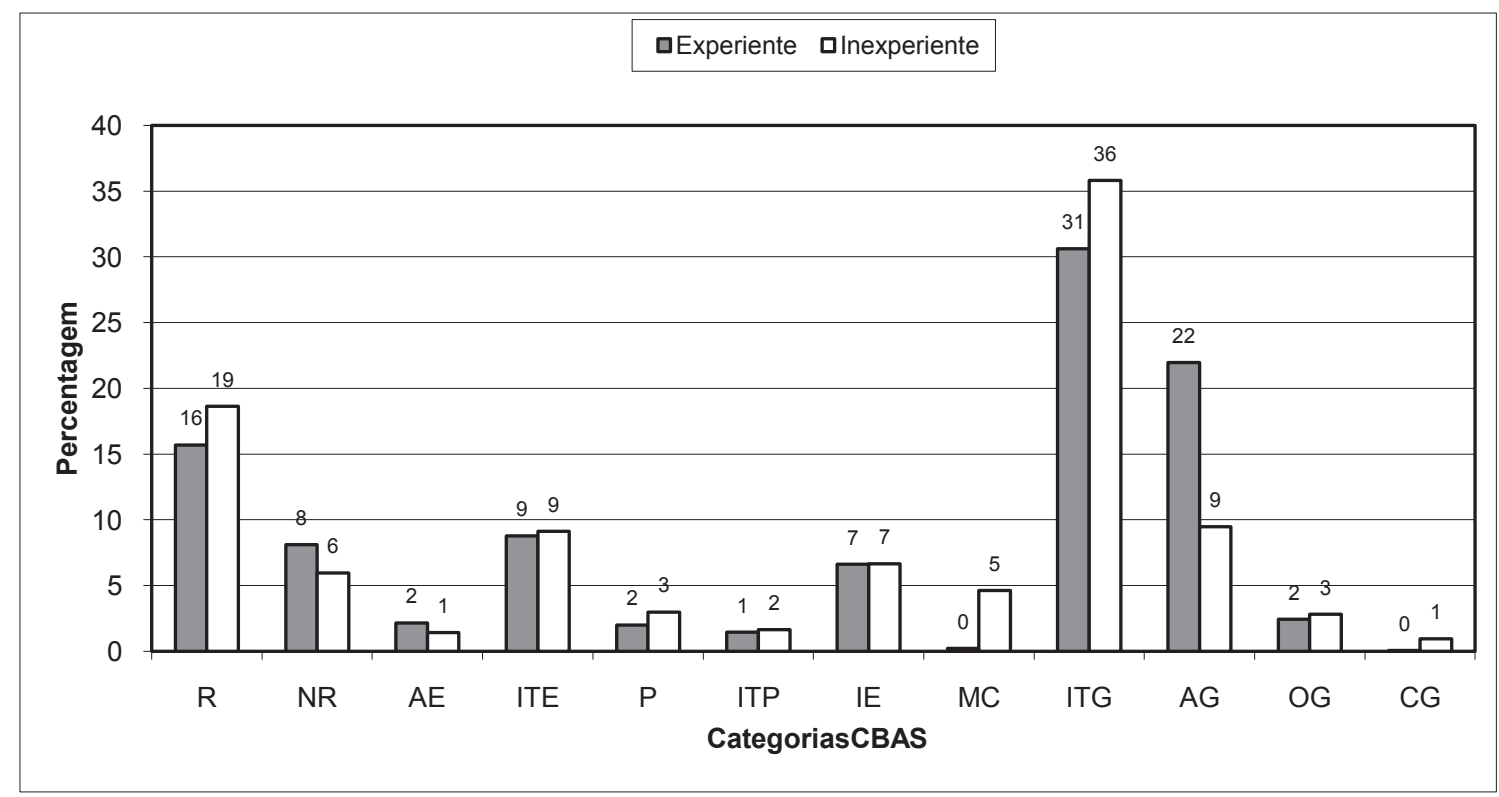

FIGURA 2 - Percentagem de ocorrências nas categorias do Coach Behavior Assessment System/CBAS para cada um dos grupos de treinadores experientes e inexperientes.

A análise comparativa do perfil de intervenção dos treinadores consoante a experiência, evidencia diferenças estatísticamente significativas em quatro dos 11 domínios, a saber: instrução técnica punitiva $(\mathrm{t}$ $=3,159, \mathrm{p} \leq 0,01)$, manter o controle $(\mathrm{t}=2,623, \mathrm{p} \leq$ $0,05)$, animo geral $(\mathrm{t}=2,063, \mathrm{p} \leq 0,05)$, e comunicação $\operatorname{geral}(\mathrm{t}=3,979, \mathrm{p} \leq 0,01)$.

No que diz respeito à instrução técnica punitiva, ela é mais frequente nos treinadores inexperientes, tal como as intervençôes para manter o controle do comportamento dos jogadores ou a comunicação geral. Pelo contrário, o ânimo geral aparece como mais frequente nos comportamentos dos treinadores experientes.

\section{Atitudes}

Dos 23 itens do questionário, existem 10 (itens 1 , $4,6,8,11,12,15,17,21,22)$ em que a concordância é socialmente desejável ( 4 ou 5 na escala de 1 a 5 ). Nesses itens, por duas vezes são observadas diferenças 
estatisticamente significativas (itens 17 e 22). O grupo supervisionado por treinadores experientes responde sempre com valores de concordância superiores.

Existem 13 itens em que a resposta socialmente desejável seria de discordância, isto é, 1 ou 2 na escala de 1 a 5 . Por seis vezes (itens 2, 3, 7, 18, 20, 23) as diferenças são estatisticamente significativas, com o grupo enquadrado por treinadores inexperientes a revelar uma posição de maior discordância. Desses seis itens, por cinco vezes (item 3 "era capaz de fazer batota se isso me ajudasse a ganhar", item 7 "como não é contra as regras pressionar psicologicamente os adversários, posso fazê-lo", item 18 "se não quiser que alguém jogue bem, tento perturbá-lo um pouco", item 20 "é uma boa ideia irritar os meus adversários", item 23 "tento que os árbitros decidam a meu favor, mesmo que não seja verdade”) a média do grupo supervisionado por treinadores inexperientes é de discordância, enquanto os seus pares orientados por treinadores mais experientes respondem com resultados acima de 3,00. Os resultados sugerem que os praticantes dos treinadores experientes estão mais predispostos a respeitar as regras do jogo e a manter um nível ligeiramente superior de empenhamento, mas também mais abertos a fazer algo mais para chegar à vitória.

A estatística descritiva das dimensões extraídas do SAQp e f-testes decorrentes da análise multivariada da variância para testar o efeito da experiência do treinador estão patentes na TABELA 3.

TABELA 3 - Estatística descritiva e ANOVA para testar o efeito da experiência do treinador sobre as dimensões do Sports Attitudes Questionnaire (SAQp).

\begin{tabular}{|c|c|c|c|c|c|c|c|}
\hline & \multicolumn{2}{|c|}{ Inexperiente $(n=27)$} & \multicolumn{2}{|c|}{ Experiente $(n=31)$} & \multirow{2}{*}{$\mathbf{F}$} & \multirow{2}{*}{$\mathbf{p}$} & \multirow{4}{*}{$\begin{array}{l}\text { n.s. (não significativo) } \\
{ }^{*}(p \leq 0,05) \\
* * \\
*(p \leq 0,01) .\end{array}$} \\
\hline & Média & DP & Média & DP & & & \\
\hline Batota & 1,88 & 0,98 & 2,50 & 1,01 & 5,587 & $*$ & \\
\hline Antidesportivismo & 2,50 & 0,90 & 3,27 & 1,08 & 8,453 & $* *$ & \\
\hline Convenção & 4,45 & 0,53 & 4,73 & 0,40 & 5,233 & $*$ & \\
\hline Compromisso & 4,33 & 0,58 & 4,36 & 0,39 & 0,053 & n.s. $[0,82]$ & \\
\hline
\end{tabular}

Quando analisamos o efeito da experiência do treinador sobre as dimensões extraídas do SAQp, observa-se um efeito estatisticamente significativo $\left[\right.$ Lambda de Wilks $=0,764, \mathrm{~F}_{(4,53)}=4,101, \mathrm{p} \leq$ $0,01]$, em que a significância da prova multivariada é atribuível aos fatores batota, anti-desportivismo e convenção. Para todas as variáveis dependentes, os jogadores sob orientação de treinadores experientes revelam pontuações médias superiores. No caso da batota, tal significa uma demonstração de menor discordância (embora, em ambos os casos, a média de respostas seja inferior a 3,00) e, no caso do antidesportivismo, de certa concordância, ao contrário dos seus pares que treinam sob a supervisão de treinadores inexperientes (uma vez que a média das respostas deste último grupo se situa abaixo de 3,00, enquanto que a média do outro grupo está acima de 3,00). De realçar que a menor discordância dos atletas treinados por treinadores experientes relativamente ao desportivismo, é correlato de maior concordância face ao respeito pelas convençôes sociais.

\section{Orientação para a realização de objetivos}

Entre os 13 itens do TEOSQp, apenas por uma vez foi observada a existência de diferenças estatísticamente significativas, no item 12 "sinto que uma técnica que aprendo está bem” ( $\mathrm{t}=2,253, \mathrm{p} \leq 0,05)$. Este resultado pode ser atribuível ao acaso, não tendo correspondência na análise multivariada dos efeitos da experiência do treinador sobre as dimensões latentes do instrumento. $\mathrm{O}$ efeito da experiência do treinador sobre as dimensões do TEOSQp não é significativo.

$\mathrm{Na}$ TABELA 4, apresentam-se os resultados da estatística descritiva das dimensões extraídas do TEOSQp e F-testes decorrentes da análise multivariada da variância para testar o efeito da experiência do treinador. Verifica-se que nem a dimensão tarefa nem a dimensão ego se mostram sensíveis ao efeito da experiência do treinador (Lambda de Wilks = 0,995, $\left.\mathrm{F}_{(2,58)}=0,153, \mathrm{p}=0,86\right)$, confirmando as evidências resultantes da exploração dos itens do questionário e questionando os estudos referidos supra. 
TABELA 4 - Estatística descritiva e ANOVA para testar o efeito da experiência do treinador sobre as dimensões do Task and Ego Orientation in Sport Questionnaire (TEOSQp).

n.s. (não significativo)

${ }^{*}(p \leq 0,05)$;

$* *(p \leq 0,01)$ n.s. (não significativo):

$*(p \leq 0,05)$;

$* *(p \leq 0,01)$

\begin{tabular}{lcccccc}
\hline & \multicolumn{2}{c}{ Inexperiente $(\mathbf{n}=\mathbf{3 0})$} & \multicolumn{2}{c}{ Experiente $(\mathbf{n}=\mathbf{3 1})$} & \multirow{2}{*}{$\mathbf{F}$} & \multirow{2}{*}{$\mathbf{p}$} \\
\cline { 2 - 5 } & Média & $\mathbf{D P}$ & Média & DP & & n.s. \\
\hline Tarefa & 3,46 & 0,49 & 3,44 & 0,43 & 0,016 & n.s. \\
Ego & 1,90 & 0,69 & 1,81 & 0,65 & 0,300 & . \\
\hline
\end{tabular}

Coach Behavior Assessment System/CBAS

Na TABELA 5 é apresentada a estatística descritiva das dimensões extraídas do CBASp e provas $\mathrm{t}$ decorrentes da análise multivariada da variância para testar o efeito da experiência do treinador. $\mathrm{O}$ estudo das respostas proporcionadas pelo CBAS, revela que o efeito da variável independente - experiência ou inexperiência do treinador - é significativo em seis dos 11 itens, sendo de notar que em outros três itens o nível de significância se situa até 0,11 . Os jogadores que desenvolvem a sua prática desportiva sob a orientação de um treinador experiente são mais vezes lembrados das táticas que devem seguir (item $1, \mathrm{t}=$ $-2,134, \mathrm{p} \leq 0,05)$, são mais vezes animados quando cometem um erro (item $3, \mathrm{t}=-2,293, \mathrm{p} \leq 0,05$ ), são mais vezes informados sobre as correções necessárias para evitar novos erros (item $4, \mathrm{t}=-3,290, \mathrm{p} \leq 0,01$ ), vêem os seus erros ignorados com menor frequência (item $7, \mathrm{t}=2,038, \mathrm{p} \leq 0,05$ ), são menos vezes distraídos pelas intervençôes do treinador (item 10, $\mathrm{t}=2,091, \mathrm{p} \leq 0,05)$, e recebem mais vezes instruçôes táticas durante os episódios de substituições (item 11, $\mathrm{t}=-3,411, \mathrm{p} \leq 0,01)$.

TABELA 5 - Estatística descritiva e teste $t$ de amostras independentes para testar o efeito da experiência do treinador sobre os itens do Coach Behaviour Assessment System (CBASp).

\begin{tabular}{|c|c|c|c|c|c|c|c|}
\hline & & \multicolumn{2}{|c|}{$\begin{array}{l}\text { Inexperiente } \\
\quad(\mathbf{n}=\mathbf{2 7})\end{array}$} & \multicolumn{2}{|c|}{$\begin{array}{l}\text { Experiente } \\
(\mathbf{n}=31)\end{array}$} & \multirow[t]{2}{*}{$F$} & \multirow[t]{2}{*}{$\mathbf{p}$} \\
\hline & & Média & DP & Média & DP & & \\
\hline 1 & $\begin{array}{l}\text { Vai-te lembrando as diferentes táticas que deves } \\
\text { seguir? (por exemplo: como defender, como } \\
\text { atacar, como te deves colocar...) }\end{array}$ & 3,78 & 1,12 & 4,29 & 0,59 & $-2,134$ & * \\
\hline 2 & $\begin{array}{l}\text { Felicita-te quando fazes uma "boa jogada"? (por } \\
\text { exemplo: roubar uma bola, marcar um cesto...) }\end{array}$ & 4,19 & 0,88 & 4,29 & 0,56 & $-0,527$ & n.s. \\
\hline 3 & $\begin{array}{l}\text { Anima-te quando cometes um erro? (por } \\
\text { exemplo: quando perdes uma bola, quando fazes } \\
\text { "passos"...) }\end{array}$ & 3,11 & 1,16 & 3,71 & 0,82 & $-2,293$ & $*$ \\
\hline 4 & $\begin{array}{l}\text { Quando cometes um erro explica-te como tens de } \\
\text { o corrigir? }\end{array}$ & 3,48 & 1,16 & 4,32 & 0,70 & $-3,290$ & ** \\
\hline 5 & $\begin{array}{l}\text { Zanga-se quando cometes um erro? (por exemplo: } \\
\text { ralha-te, substitui-te...) }\end{array}$ & 3,04 & 1,13 & 2,61 & 0,88 & 1,607 & n.s. \\
\hline 6 & $\begin{array}{l}\text { Quando dá uma instrução após um erro, fá-lo a } \\
\text { gritar ou a ralhar? }\end{array}$ & 2,78 & 1,05 & 2,39 & 0,67 & 1,663 & n.s. \\
\hline 7 & $\begin{array}{l}\text { Ignora os erros que cometes? (não faz comentá- } \\
\text { rios, nem positivos, nem negativos) }\end{array}$ & 2,30 & 1,07 & 1,77 & 0,88 & 2,038 & $*$ \\
\hline 8 & $\begin{array}{l}\text { Se não estás atento, chama-te a atenção para que } \\
\text { escutes as suas instruçôes? }\end{array}$ & 3,93 & 0,92 & 4,32 & 0,75 & $-1,814$ & n.s. \\
\hline 9 & Anima-te? & 3,70 & 0,99 & 3,87 & 0,89 & $-0,678$ & n.s. \\
\hline 10 & $\begin{array}{l}\text { Distrai-se com facilidade? (por exemplo: fala de } \\
\text { coisas que não têm a ver com o jogo ou com o } \\
\text { treino) }\end{array}$ & 2,04 & 0,94 & 1,58 & 0,72 & 2,091 & $*$ \\
\hline 11 & $\begin{array}{l}\text { Quando faz substituiçôes, dá-te instruçōes sobre a } \\
\text { tática a seguir? }\end{array}$ & 3,59 & 1,12 & 4,42 & 0,62 & $-3,411$ & ** \\
\hline
\end{tabular}

22 • Rev. bras. Educ. Fís. Esporte, São Paulo, v.24, n.1, p.15-26, jan./mar. 2010 


\section{Discussão}

As percepções mútuas do treinador e do atleta $\mathrm{e}$ o seu relacionamento representam um papel crucial na motivação para a prática e proporcionam maior satisfação na atividade (LORIMER \& JOWETT, 2009). Estudos realizados tanto com atletas de elite como os atletas adolescentes (Fraser-ThOMAS \& CÔTÉ, 2009; JaCKSON, KNAPP \& BEAUCHAMP, 2009) evidenciam a importância dessa interação, quando centrada em ambiente orientado para a Tarefa, bem como as experiências desportivas, que podem ser positivas ou negativas, em função da ecologia de prática resultante em boa parte da intervenção do treinador.

A experiência desportiva parece dispensar uma maior necessidade de intervir para controlar a disciplina e punir, sendo valorizada a importância de manter o estado anímico favorável durante a competição. Enquanto o treinador inexperiente parece preocupado com a eclosão de comportamentos desviantes, o treinador experiente privilegia a indução de um estado de ativação para a competição.

A presente análise baseia-se na ponderação de frequências de intervenções, não se devendo inferir que o impacto emocional de um episódio de Reforço seja equivalente ao que resulta de Instrução Técnica Punitiva ou de Punição, menos frequentes mas potencialmente causadores de maior perturbação para o jovem atleta. A menor frequência de certas intervençōes negativas do treinador não deve mascarar o impacto que possam vir a ter.

Seria plausível que a ação do treinador, como elemento ecológico importante da prática desportiva, não teria um efeito nulo sobre as orientaçôes para a realização de objetivos, tendo em conta estudos precedentes (Kavussanu \& NTOUMANIS, 2003; Kavussanu \& Spray, 2006; Sage $\&$ Kavussanu, 2007; Sage, Kavussanu \& Duda, 2006). Por outro lado, o efeito da experiência do treinador sobre as atitudes é significativo no que diz respeito à batota, antidesportivismo e convençãa. Estes resultados sugerem que existem outros fatores que podem funcionar como mediadores na expressão de orientações para a realização de objetivos por parte dos atletas e a necessitar de outras investigaçōes.

Os resultados sugerem que o treinador experiente está mais atento aos aspectos técnico-táticos do jogo e confere maior importância às intervenções contextualizadas e imediatamente resultantes das ações dos jogadores. A experiência parece permitir ao treinador uma maior prontidão para intervir no jogo e melhor reatividade às necessidades de apoio e encorajamento dos atletas. Todavia, em estudo com treinadores de Futebol do escalão de sub-16, na Catalunha, utilizando idêntica metodologia SousA et al. (2006) mostram que os atletas dos treinadores com menos experiência percepcionam um menor conhecimento do jogo dos seus treinadores, mas maior apoio e encorajamento, face aos seus pares orientados por treinadores mais experientes. Os mesmos autores sugerem que as diferenças entre os treinadores se localizam menos na sua experiência de treino do que na biografia do treinador e na sua formação pessoal e desportiva, propondo uma abordagem individualizada do treinador em futuras intervenções com caráter formativo.

Do mesmo modo, os resultados sugerem que as instruções dos treinadores com maior experiência possam ser mais complexas e que o ânimo seja proporcionado aos jogadores de modo mais adequado. Mas deve ser tido em conta que o CBAS capta apenas a frequência de episódios de comunicação que ocorrem durante o jogo e não a qualidade da interação do treinador com os jogadores, devendo ser complementado por outras metodologias, nomeadamente qualitativas.

De acordo com Schempr, McCullick e Sannen MASON (2006) parte-se do princípio que o tempo representa um fator de aprendizagem para o treinador e que após vários anos de experiência se vão produzir melhorias na sua intervenção, tendo em conta que uma parte importante da sua formação é realizada através da prática do treino. Permanecem por compreender os mecanismos que sustentam a aprendizagem prática e de que modo ela é recepcionada de modo diferenciado pelos treinadores com diversas biografias e que exercem a sua atividade em ambientes ecológicos distintos.

Feltz, Hepler e Paiement (2009), em estudo com treinadores voluntários de jovens, referem que a experiência anterior como atleta ou treinador vai influenciar a auto-confiança do treinador e consequentemente as percepçōes positivas sobre o desenvolvimento e comportamento dos seus atletas, proporcionando condiçóes para um clima de treino orientado para a Tarefa e para uma melhor relação com os praticantes.

Em estudos anteriores (Jones \& STANDAGE, 2006; Jones \& Wallace, 2006; Olympiou, Jowett \& DUDA, 2008) as amostras de treinadores foram reduzidas e de conveniência, focando essencialmente o discurso do treinador com anos de prática e sucesso competitivo nos níveis máximos de rendimento. Os resultados, embora apontando as diferenças entre as Atitudes face à Prática Desportiva e as Percepçôes 
do Comportamento do Treinador dos atletas inquiridos, sugerem que a exploração do tema do impacto educativo e moral da comunicação do treinador seja realizada, tal como propõe CRUZ (2003), ao nível individual, do treinador e da equipe, tendo em conta o ambiente ecológico em que a prática desportiva tem lugar.

\title{
Conclusões
}

O estudo pretendeu apontar a importância que a experiência profissional do treinador de jovens representa na formatação do tipo de relacionamento e de comunicação que é privilegiado nas interações com os atletas. A importância do conhecimento da realidade da intervenção do treinador no desporto infanto-juvenil poderá ter consequências para a formação inicial dos treinadores, para ações de formação contínua ou tutoriais específicas com treinadores experientes. $\mathrm{O}$ percurso pessoal como atleta e como treinador parece marcar o estilo de intervenção. A investigação deve ter em conta a exploração das várias ecologias de prática que incluam todas as dimensões que marcam o relacionamento complexo do treinador com o atleta. Para além do estudo de outros contextos e de outros universos amostrais, parece importante recorrer complementarmente a metodologias qualitativas, face aos limites de um estudo observacional e quantitativo, que não ilumina com clareza a qualidade das interaçōes desportivas e pessoais.

Do mesmo modo, parece que a formação do treinador de jovens deve ter em conta o processo de aprendizagem contínua do treinador e a sua dimensão incontornavelmente pedagógica. Todavia a maioria dos curriculuns de formação de treinadores não confere uma importância decisiva a estas questões, secundárias em relação aos aspectos técnicotáticos. O contato com a investigação e a interação com as organizações desportivas deveriam fomentar o interesse dos treinadores pelo tópico, como meio de melhorar a qualidade da sua intervenção.

\begin{abstract}
The effects of coaches' experience on the motivational and pedagogical climate in youth sport

The study analyzes the effects of the coaches' professional experience on the coach-athlete communication during competitions, achievement goal orientations, sport attitudes and athletes' perceptions of the coaches'behaviour. Using the Coach Behaviour Assessment System/CBAS (SмiтH, SMoll \& Hunt, 1977), 18 games of six Basketball coaches (three of them experiencent and three unexperient) of male under-16 years teams were audio and video recorded and subsequently analyzed. The players of the observed teams $(n=58)$ fulfilled the portuguese versions of the Task and Ego Orientation in Sport Questionnaire/ TEOSO (CHI \& DUdA, 1995), the Sport Attitudes Questionnaire/SAO (LEE \& WHITEHEAD, 1999), and of the CBAS questionnaire about the perceptions of the coaches' behaviour. The results show a statistically significant effect of the coaches' experience both on the behaviour categories observed during competition and on the questionnaires' dimensions.
\end{abstract}

UnIterms: Youth; Coach; Task; Ego; Attitudes.

\section{Referências}

BOIXADÓS, M.; CRUZ, J.; TORREGROSA, M.; VALIENTE, L. Relationships among motivational climate, satisfaction, perceived ability, and fair play attitudes in young soccer players. Journal of Applied Sport Psychology, Chapel Hill, v.16, p.301-17, 2004. 
BRUSTAD, R.; PARTRIDGE, J. Parental and peer influence on children's psychosocial development through sport. In: SMOLL, F.; SMITH, R. (Orgs.). Children and youth in sport: a biopsychosocial perspective. Dubuque: Kendall/Hunt, 2002.

CHI, L.; DUDA, J. Multi-sample confirmatory factor analysis of the task and ego orientation in sport questionnaire. Research Quarterly for Exercise and Sport, Washington, v.66, n.2, p.91-8, 1995.

COELHO E SILVA, M.; ROCHA, I.; GONÇALVES, C.E. Treinador de jovens e formação de jovens treinadores. In: COELHO E SILVA, M.; GONÇALVES, C.E.; FIGUEIREDO, A. (Orgs.). Desporto de jovens ou jovens no desporto? Coimbra: Faculdade de Ciências do Desporto e Educação Física, 2006.

COHEN, J.A. Coefficient of agreement for nominal scales. Educational Psychological Measurement, New York, v.20, p.36-7, 1960. CRUZ, J. Factores motivacionales en el deporte infantil y asesoriamento psicológico a entrenadores y padres. In: CRUZ FELIU, J. (Orgs.). Psicologia del deporte. Madrid: Sintesis, 2001.

Asesoramiento psicologico a entrenadores de deportistas jóvenes. In: OÑA SICILIA, A.; BILBAO GUERRERO,

A. (Orgs.). Proceedings of the II World Congress of Physical Activity and Sport Sciences: Sport and Quality of Life. Granada: Alhambra, 2003.

CRUZ, J.; BOIXADÓS, M.; VALIENTE, L.; TORREGROSA, M.; MIMBRERO, J. Existe un deporte educativo?: papel de las competiciones deportivas en el proceso de socialización del niño. Revista de Psicologia del Deporte, Palma de Maiorca, v.9/10, p.111-32, 1996.

DUDA, J. Achievement goal research in sport: pushing the boundaries and clarifying some misunderstandings. In: ROBERTS, G. (Org.). Advances in motivation in sport and exercise. Champaign: Human Kinetics, 2001.

FELTZ, D.; HEPLER, T.; ROMAN, N.; PAIEMENT, C. Coach efficacy and volunteer youth sport coaches. The Sport Psychologist, Champaign, v.23, p.24-41, 2009.

FONSECA, A.M.; BIDDLE, S.J. Estudo inicial para a adaptação do task and ego orientation in sport questionnaire (TEOSQ) à realidade portuguesa. In: INTERNATIONAL CONFERENCE ON PSYCHOLOGICAL ASSESSMENT: Development and Contexts, 4., 1996, Braga. Actas... Braga: Imprensa da Universidade do Minho, 1996.

FRASER-THOMAS, J.; CÔTÉ, J. Understanding adolescents' positive and negative developmental experiences in sport. The Sport Psychologist, Champaign, v.23, p.3-23, 2009.

GILBERT, W.; TRUDEL, P. Role of the coach: how model youth team sport coachesframe their roles. The Sport Psychologist, Champaign, v.18, p.21-43, 2004.

GONÇALVES, C.E.; CARDOSO, L.; FREITAS, F.; LOURENÇO, J.; COELHO E SILVA, M. Valores no desporto de jovens: concepções, instrumentos e limitaçôes. Boletim da Sociedade Portuguesa de Educação Física, Lisboa, v.30/31, p.93-110, 2005. GONÇALVES, C.E.; COELHO E SILVA, M.; CRUZ, J. Efeitos do nível desportivo sobre as atitudes face à prática desportiva e a orientação para a realização de objectivos em jovens atletas dos 13 aos 16 anos. In: COELHO E SILVA, M.; GONÇALVES, C.E.; FIGUEIREDO, A. (Orgs.). Desporto de jovens ou jovens no desporto? Coimbra: FCDEF-UC, 2006. GONÇALVES, C.E.; COELHO E SILVA, M.; CRUZ, J.; LEE, M.J.; CHATZISARANTIS, N. Tradução e validação do sports attitudes questionnaire para atletas portugueses dos 13-16 anos. Revista Portuguesa de Ciências do Desporto, Porto, v.6, n.1, p.38-49, 2006.

GREENDORFER, S.; LEWKO, J.; ROSENGREN, K. Family and gender-based influences in sport socialization of children and adolescents. In: SMOLL, F.; SMITH, R. (Orgs.). Children and youth in sport: a biopsychosocial perspective. Dubuque: Kendall/Hunt, 2002.

HANRAHAN, S.; BIDDLE, S. Measurement of achievement orientations: psychometric measures, gender, and sport differences. European Journal of Sport Science, Cologne, v.2, n.5, p.20-32, 2002.

JACKSON, B.; KNAPP, P.; BEAUCHAMP, M. The coach-athlete relationship: a tripartite efficacy perspective. The Sport Psychologist, Champaign, v.23, p.203-32, 2009.

JONES, R.; STANDAGE, M. First among equals: shared leadership in the coaching context. In: JONES, R. (Org.). The sports coach as an educator: re-conceptualizing sports coaching. Oxon: Routledge, 2006.

JONES, R.; WALLACE, M. The coach as "orchestrator": more realistically managing the coaching context. In: JONES, R. (Org.). The sports coach as an educator: re-conceptualizing sports coaching. Oxon: Routledge, 2006.

KAVUSSANU, M.; NTOUMANIS, N. Participation in sport and moral functioning: does ego orientation mediate their relationship? Journal of Sport \& Exercise Psychology, Champaign, v.25, p.501-18, 2003.

KAVUSSANU, M.; SPRAY, C. Contextual influences on moral functioning of male youth footballers. The Sport Psychologist, Champaign, v.20, p.1-23, 2006.

LANDIS, J.R.; KOCH, G.C. The measurement of observer agreement for categorical data. Biometrics, Washington, v.33, p.159-74, 1977. 
LEE, M.J. Young people, sport and ethics: an examination of fairplay in youth sport. London: Sports Council Research Unit, London, 1996. (Unpublished report).

LEE, M.J.; WHITEHEAD, J. The effects of values, achievement goals, and perceived ability on moral attitudes in youth sport. Swindon: Economic and Social Research Council, 1999. (Report).

LEE, M.J.; WHITEHEAD, J.; BALCHIN, N. The measurement of values in youth sport: development of the youth sport values questionnaire. Journal of Sport and Exercise Psychology, Champaign, v.22, p.307-26, 2000.

LORIMER, R.; JOWETT, S. Empathic accuracy, metaperspective, and satisfaction in the coach-athlete relationship. Journal of Applied Sport Psychology, Chapel Hill, v.21, p.204-12, 2009.

MAGYAR, T.M.; FELZ, D. The influence of dispositional and situational tendencies on adolescent girls' sport confidence sources. Psychology of Sport \& Exercise, Amsterdan, v.4, p.175-90, 2003.

NICHOLLS, J.G. The competitive ethos and democratic education. Cambridge: Harvard University Press, 1989.

NTOUMANIS, N.; VAZOU, S. Peer motivational climate in youth sport: measurement development and validation. Journal of Sport \& Exercise Psychology, Champaign, v.27, p.432-55, 2005.

OLYMPIOU, A.; JOWETT, S.; DUDA, J. The psychological interface between the coach-created motivational climate and the coach-athlete relationship in team sports. The Sport Psychologist, Champaign, v.22. p.423-38, 2008.

PEIRÓ, C.; SANCHÍS, J.M. Las propriedades psicométricas de la versión inicial del cuestionario de orientación a la tarea y al ego (TEOSQ) adaptado a la educación física en su traducción al castellano. Revista de Psicologia del Deporte, Palma de Maiorca, v.13, n.1, p.26-40, 2004.

PENNEY, D. Coaching as teaching: new aknowledgements in practice. In: JONES, R. (Org.). The sports coach as an educator: re-conceptualizing sports coaching. Oxon: Routledge, 2006.

ROBERTS, G. Understanding the dynamics of motivation in physical activity: the influence of achievement goals on motivational process. In: ROBERTS, G. (Org.). Advances in motivation in sport and exercise. Champaign: Human Kinetics, 2001. SAGE, L.; KAVUSSANU, M. The effects of goal involvement on moral behavior in an experimental manipulated competitive setting. Journal of Sport \& Exercise Psychology, Champaign, v.29, n.2, p.190-207, 2007.

SAGE, L.; KAVUSSANU, M.; DUDA J. Goal orientations and moral identity as predictors of prosocial and antisocial functioning in male association football players. Journal of Sports Sciences, London, v.24, n.5, p.455-66, 2006.

SCHEMPP, P.; McCULLICK, B.; SANNEN MASON, I. The development of expert coaching. In: JONES, R. (Org.). The sports coach as an educator: re-conceptualizing sports coaching. Oxon: Routledge, 2006.

SMITH, R.; SMOLL, F.; HUNT, E. A system for the behavioral assessment of athletic coaches. Research Quarterly, Washington, v.48, p.401-7, 1977.

SMOLL, F.; SMITH, R. Coaching behavior research and intervention in youth sports. In: SMOLL, F; SMITH, R. (Orgs.). Children and youth in sport: a biopsychosocial perspective. Dubuque: Kendall/Hunt, 2002.

SOUSA, C.; CRUZ, J.; TORREGROSA, M.; VILCHES, D.; VILADRICH, C. Evaluación conductual y programa de asesoramiento personalizado a entrenadores (PAPE) de deportistas. Revista de Psicología del Deporte, Palma de Maiorca, v.15, n.2, p.263-78, 2006.

TREASURE, D. Enhancing young people's motivation in youth sport: an achievement goal approach. In: ROBERTS, G. (Org.). Advances in motivation in sport and exercise. Champaign: Human Kinetics, 2001.

VALLERAND, R.; DESHAIES, P.; COURIER, J.P.; BRIÈRE, N.; PELLETIER, L. Toward a multidimensional definition of sportsmanship. Journal of Applied Sport Psychology, Chapel Hill, v.8, p.89-101, 1996.

VAZOU, S.; NTOUMANIS, N.; DUDA, J. Peer motivational climate in youth sport: a qualitative inquiry. Psychology of Sport \& Exercise, Amsterdan, v.6, p.497-516, 2005.

WEIGAND, D.; CARR, S.; PETHERICK, C.; TAYLOR, A. Motivational climate in sport and physical education: the role of significant others. European Journal of Sport Science, Cologne, v.1, n.4, p.1-13, 2001.

WERTHNER, P.; TRUDEL, P. A new theoretical perspective for understanding how coaches learn to coach. The Sport Psychologist, Champaign, v.20, p.198-212, 2006.

ENDEREÇO

Carlos Eduardo Gonçalves

Faculdade de Ciências do Desporto e Educação Física Estádio Universitário - Pavilhão III 3040-156 - Coimbra - PORTUGAL e-mail: carlosgoncalves@fcdef.uc.pt

Recebido para publicação: 20/08/2008

1a. Revisão: 17/06/2009

2a. Revisão: 03/08/2009

3a. Revisão: 12/08/2009

Aceito: 04/09/2009

26 - Rev. bras. Educ. Fís. Esporte, São Paulo, v.24, n.1, p.15-26, jan./mar. 2010 